and the result follows as before. A calculation gives the zero $1.272887+.0649729 i$ for $B_{6}(s)$.

\title{
REFERENCE
}

1. N. E. Nörlund, Mémoire sur les polynomes de Bernoulli, Acta Math. 43 (1920), 121-131.

University of Tennessee

\section{DIRECTED UNIONS AND CHAINS}

JOHN R. ISBELL

It is a well known consequence of the axiom of choice that forming unions of directed families can be reduced to (repeatedly) forming unions of chains. The most general formulation of this fact seems to be P. M. Cohn's [1, p. 33]: in a partially ordered set, if every wellordered subset has a least upper bound, then every directed subset has a least upper bound. This note is mainly concerned with proving Cohn's result without the axiom of choice, but assuming the directed subset has an ordinal cardinal number. (Cohn's use of the axiom goes well beyond that.) Incidentally, well-ordered sets of the same size suffice.

It seems to be still unknown whether there exist models for set theory in which $\omega_{1}$ is the limit of a countable sequence of countable ordinals. In any such model, of course, when countable sequences have suprema, $\omega_{1}$ sequences have suprema.

Theorem. Let $P$ be a partially ordered set and $\omega_{\alpha}$ an initial ordinal such that every chain in $P$ of order type $\leqq \omega_{\alpha}$ has a supremum in $P$. Then every directed subset of $P$ of power at most $\boldsymbol{\aleph}_{\alpha}$ has a supremum.

Proof. Let $D$ be a directed subset whose elements $x_{\beta}$ are indexed by ordinals $\beta<\omega_{\alpha}$. For each index $\beta$ and finite set $F$ of indices, we define $z(\beta, F)$ by recursion on $\beta$. Let $D(F)$ be the subset of $D$ indexed by $F$. Let $z(0, F)$ be the first-indexed $x_{\gamma}$ that is a common successor of the elements of $D(F)$. If $\beta=\lambda+k$ where $\lambda$ is a limit ordinal (possibly 0 ) and $k$ is finite nonzero, $z(\beta, F)=z(\lambda, F \cup G)$ where $G$ is the set $\{\lambda, \lambda+1, \cdots, \lambda+k-1\}$. We shall show that $z(\beta, F)$ is monotonic in

Received by the editors October 21, 1965. 
$\beta$, and define $z(\lambda, F)$ for a nonzero limit ordinal $\lambda$ as the supremum of the preceding chain $\{z(\beta, F): \beta<\lambda\}$. Evidently it will suffice to show $z(\beta+1, F) \geqq z(\beta, F)$ for $\beta<\lambda$. This holds if $z(\mu, G)$ is monotonic in $G$ for the largest limit ordinal $\mu \leqq \beta$. The first ordinal $\nu$ for which $z(\nu, G)$ is not monotonic in $G$ is not 0 ; not a successor ordinal; not a limit ordinal at which $z(\nu, G)$ is the supremum of $\{z(\beta, G): \beta<\nu\}$. So $z(\beta, F)$ is defined for all $\beta$ and $F$. In particular, we have a chain $\{z(\beta, \Lambda)\}$ for the null set $\Lambda$ which has a supremum $p$ in $P$. Since $z(\beta+1, \Lambda) \geqq x_{\beta}, p$ is an upper bound of $D$. Any upper bound $q$ of $D$ exceeds all $z(0, F)$, and by induction $q$ exceeds all $z(\lambda+k, F)$ (or $z(\lambda, F)$ ) when this holds for the predecessors of $\lambda+k$ (or $\lambda$ ). Thus $q$ must exceed $p$, which is the required supremum.

No such theorem holds if "supremum" is replaced by "upper bound." To see this, take any directed set $D$ having no cofinal chain and attach an element $\gamma$ for each chain $C$ in $D$, with no order relations except those in $D$ and $\gamma>d$ whenever there is $c$ in $C$ such that $c \geqq d$.

Assuming the axiom of choice, a related bit of information can be added. The lower character of a non trivial directed set (i.e. one lacking a finite cofinal subset) is the smallest power of an unbounded subset; it is an invariant of cofinal type, and a rather useful one [2].

Proposition. The lower character of a directed set is the smallest power of an unbounded chain in it.

Proof. Given an unbounded subset $\left\{x_{\beta}\right\}$ of smallest power $\boldsymbol{\aleph}_{\alpha}$, indexed by ordinals $\beta<\omega_{\alpha}$, choose $z_{\beta}$ recursively as a bound for the set consisting of $x_{\beta}$ and all preceding $z_{\gamma}$. The $z_{\beta}$ form the required chain.

\section{REFERENCES}

1. P. M. Cohn, Universal algebra, Harper and Row, New York, 1965.

2. J. R. Isbell, The category of cofinal types. II, Trans. Amer. Math. Soc. 116 (1965), 394-416.

Case Institute of Technology 